\title{
Botulinum toxin A in the treatment of Raynaud's phenomenon: a systematic review
}

\author{
Paweł Żebryk, Mariusz J. Puszczewicz
}

Department of Rheumatology and Internal Medicine, Poznan University of Medical Sciences, Poznan, Poland

Submitted: 29 July 2014

Accepted: 13 August 2014

Arch Med Sci 2016; 12, 4: 864-870

DOI: $10.5114 /$ aoms.2015.48152

Copyright $\odot 2015$ Termedia \& Banach

\section{Abstract}

Introduction: The management of Raynaud's phenomenon in its most severe form is challenging, and current medical and surgical treatment methods frequently do not lead to optimal symptom control and prevention of ischemic complications. The aim of the study was to critically evaluate all existing evidence on the use of botulinum toxin $A$ in the management of Raynaud's phenomenon.

Material and methods: We adopted the PRISMA methodology and searched Cochrane Library, MEDLINE, SCOPUS, EULAR and ACR congresses abstract archives for Raynaud* AND botulinum toxin OR onabotulinum. All studies that contained reports of botulinum toxin A use and its outcome in Raynaud's phenomenon were included in the review.

Results: Eleven studies met our inclusion criteria and involved a total of 125 patients. Two reviewers extracted data from the studies under review and achieved a consensus in their selection. The main outcomes measured were pain reduction and healing of digital ulcers. The level of evidence across studies was very low to moderate.

Conclusions: There is insufficient evidence to assess the efficacy of botulinum toxin A in Raynaud's phenomenon. Despite many promising reports, further research in the form of randomized controlled trials is warranted in order to investigate this new treatment method for Raynaud's phenomenon.

Key words: botulinum toxins, type A/therapeutic use, Raynaud disease/ etiology/therapy, scleroderma, systemic/*therapy, vasodilator agents/ therapeutic use.

\section{Introduction}

Raynaud's phenomenon (RP) is one of the common causes of patient consultation with a rheumatologist. It is thought to be an exaggerated physiological response of blood vessels in the extremities to cold and emotional stress. Classical RP involves three phases of color change from pale (vasoconstriction) then cyanotic (ischemia due to stasis of blood) to ultimately red (reactive hyperemia) [1, 2].

The prevalence of RP is estimated at around 3-5\% of the general population with a slight predilection in women [2]. The majority of patients have primary RP, which is idiopathic, usually runs a mild course and by definition is not associated with an underlying connective tissue disease (CTD) $[1,3]$. The prevalence of primary RP exceeds that of secondary RP in the proportion of $9: 1[4]$.

\author{
Corresponding author: \\ Paweł Żebryk MD, PhD, \\ MSc ClinEd \\ Department of Rheumatology \\ and Internal Medicine \\ Poznan University \\ of Medical Sciences \\ 137/147 28 Czerwca \\ 1956 roku St \\ 61-545 Poznan, Poland \\ Phone: +48618310317 \\ E-mail: pzebryk@ump.edu.pl
}


Less frequently, RP occurs as a harbinger of systemic sclerosis (SSc) or other condition and therefore it is called secondary RP. It can lead to ulceration of the fingertips, and in extreme cases to critical ischemia and necrosis of the phalanges. Ischemic complications are frequent in SSc-related RP and never develop in primary RP. This fact could be explained by the structural vasculopathy of SSc that potentiates the effect of RP in this group of patients and therefore renders them more prone to ischemia [1].

Primary RP can be managed with lifestyle modifications: avoiding cold, minimizing stress, reducing the intake of caffeine, avoiding vasoconstrictive medication and the cessation of smoking $[1,5,6]$. Most patients with primary RP only need pharmacotherapy occasionally, in contrast to patients with secondary RP [5].

When lifestyle modifications fail, pharmacological or surgical intervention is often employed. Currently RP is managed with dihydropyridine calcium channel blockers (CCB) as first line agents, other therapies being topical glyceryl trinitrate, phosphodiesterase 5 inhibitors, a prostacyclin analogue (iloprost), an endothelin receptor antagonist (bosentan) and surgical sympathectomy [5-11].

However, in the most severe cases of RP traditional therapies frequently do not bring satisfactory results. In recent years there have been encouraging reports in the literature on the use of botulinum toxin A (BTX-A) in the treatment of RP. Several research groups have reported a significant improvement in pain reduction, RP symptom severity and healing of ulcers.

The aim of this systematic review is to analyze all available evidence on the effect of BTX-A in RP.

\section{Botulinum toxin $\mathrm{A}$}

Botulinum toxin $A$ is a neurotoxin produced by the gram-positive, spore-forming bacilli of the genus Clostridium, but mainly from strains of C. botulinum. It is a $150 \mathrm{kDa}$ protein consisting of a heavy chain and light chain connected together by a disulfide bridge. In humans BTX-A is endocytosed at the presynaptic nerve terminals and its zinc-dependent protease domain cleaves SNAP-25 (synaptosomal-associated protein 25). The loss of this protein prevents exocytosis of acetylcholine-rich presynaptic vesicles to the synaptic cleft and, therefore, abolishes signal conduction in afflicted cholinergic neurons [12].

In addition, evidence shows that BTX-A also targets noncholinergic neurons. Its inhibition of neurotransmitters such as substance P, glutamate and calcitonin-gene related peptide has been the rationale for its most recent applications in the management of chronic pain [12]. In an ex- perimental study using rodents, BTX-A was also demonstrated to block sympathetic nerve conduction and thus reduce vasoconstriction, which is the most plausible mechanism explaining its usefulness in RP [13].

Botulinum toxin A has found many cosmetic as well as medical applications since its first use in the 1990s, mainly in neurological and musculoskeletal disorders such as torticollis, migraines, headaches, detrusor overactivity, spasticity in the setting of a stroke, paraplegias and dystonias. Other uses include strabismus, blepharospasm and hyperhidrosis [14, 15].

\section{Review methods}

We adopted the PRISMA methodology for this systematic review [16]. We searched MEDLINE, COCHRANE library and SCOPUS for Raynaud* AND botulinum toxin OR onabotulinum (last time on July $\left.29^{\text {th }}, 2014\right)$. All studies that contained reports of botulinum toxin $A$ use and its outcome in RP were eligible for this review. No limitations as to language or time of publication were applied. We searched ClinicalTrials.gov and CENTRAL for clinical trials involving the use of BTX-A in RP patients. In addition, we also searched unpublished studies in meeting abstracts archives of EULAR (20012014) and ACR (2006-2013) annual congresses (last searched: July 29th 2014 ).

\section{Data synthesis}

The initial search of MEDLINE, COCHRANE, SCOPUS, EULAR and ACR abstract databases yielded 152 articles, which were narrowed down to 117 after eliminating duplicates. A careful analysis of titles and abstracts found 11 studies (including one in French) that met our inclusion criteria, of which 10 full texts were available and 1 was a conference poster. The study selection process is illustrated in the flowchart (Figure 1). A ClinicalTrials.gov and CENTRAL search of clinical trials involving the use of BTX-A in RP patients resulted in finding one completed study, one ongoing and one not yet started (as of July 29 $9^{\text {th }} 2014$ ).

Studies included in the review ranged from two single $[17,18]$ and six series of case reports [19-24], through two open label trials [25, 26] and ultimately one randomized controlled trial (RCT) [27] and involved 125 patients in total. Within this population, 38 (30\%) patients were diagnosed with primary RP and 87 (70\%) with secondary RP, 57 (46\%) of whom were diagnosed with systemic sclerosis and 6 (5\%) with mixed connective tissue disease. In terms of methodology, 4 studies were prospective $[20,25-27]$ and 7 retrospective [17-19, 21-24]. Median time from intervention to time of outcome assessment in prospective 


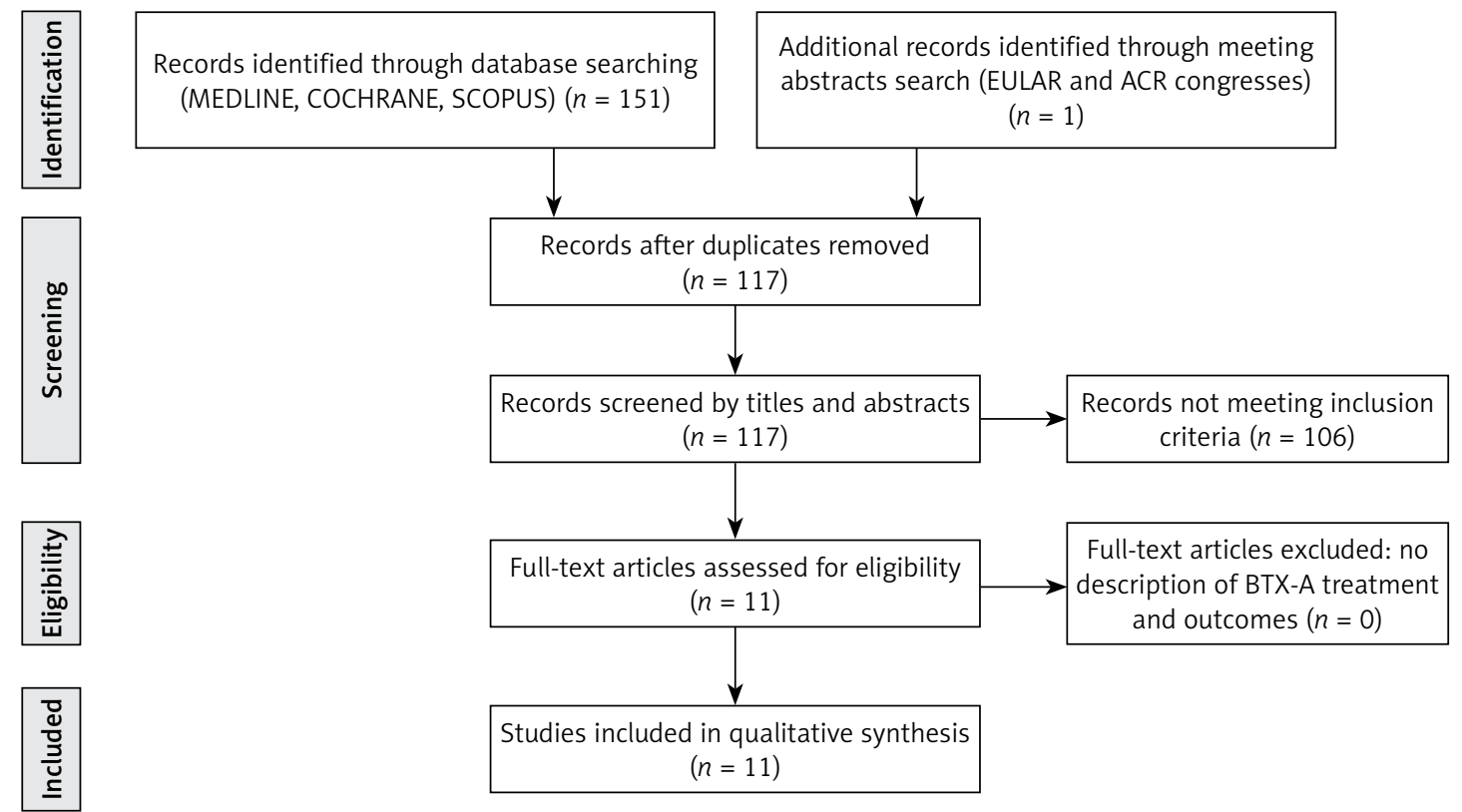

Figure 1. Results of study selection process for this systematic review

studies was 5 weeks (range 1-10) [20, 25-27]. The studies differed with respect to outcomes used, doses and sites of BTX-A injection as well as the subjects' underlying diseases. All studies are summarized in Table I.

Throughout the evaluated studies the inclusion criteria were described using different wording but all included severe symptoms of RP with or without ischemic complications not responding to conventional medical $[17,19,21-24,26]$ or surgical therapy (i.e. cervico-dorsal or digital sympathectomy) [17]. Four studies did not reveal patient drug and surgical history, while in 6 this information was partial $[19,22-24,26]$ and in 3 studies it was complete $[17,18,21]$. In 9 out of 11 studies the RP symptoms were severe enough despite currently available therapy, in 1 study BTX-A was a primary modality of treatment [18], and in an experimental study by Stadlmaier et al. this information was missing [20].

Four reports did not contain exclusion criteria [17-20], very possibly due to the retrospective design of the studies [17-19], but those which did included typical contraindications for BTX-A, namely: allergy, pregnancy, myasthenia gravis and mild RP [27] and/or vaso-occlusive disease of the extremity [22-25].

The sites of BTX-A injection were also inconsistently described across studies; however, in all but one (where the wrist was also used [22]) the study authors concentrated on neurovascular bundles of digits and/or the superficial palmar arch. Fregene et al. compared different injection sites wrist, along the neurovascular bundles of digits and the distal part of the metacarpus - and found no advantage of one over the others [22]. The tox- in was injected into selected digits [19, 22], into one hand, while the other served as a control [26, 27], or into both hands [17, 18, 20, 21, 23-25].

Different outcomes were reported in the reviewed studies, but most involved pain assessment, ulcer healing, hand perfusion measurement and function. Pain was measured using the Visual Analogue Scale (VAS) or by patient self-assessment. Perfusion was assessed either subjectively as color change or measured objectively using laser Doppler perfusion imaging (LDPI) or a thermometer. Hand perfusion and function were inconsistently described among the included studies and, therefore, difficult to compare. Nonetheless, the authors presented evidence of increased digital temperature [21, 27], improved blood flow in LDPI [17, 19, 23, 24], improvement in Disabilities of the Arm, Shoulder and Hand (DASH) score [25, 26], blood saturation [22, 25] and general improvement of RP symptoms and their frequency $[20,21]$ in patients treated with BTX-A. The efficacy of BTX-A in pain reduction or ulcer healing is difficult to assess quantitatively due to incomplete reporting of data, thus precluding a proper meta-analysis. Researchers who measured pain using the VAS report its decrease in $85-100 \%$ of patients by a mean ranging from $20 \%$ to $90 \%$ [20-22, 25, 26]. Similarly, healing of ulcers was reported in $75-100 \%$ of patients, with an average of $48-100 \%$ of ulcers healed [17, 21-26].

The BTX-A effect on a treated hand was immediate in $84 \%$ of patients according to Neumeister [23]. The duration of the effect of BTX-A in alleviating RP symptoms was on average 4 months (1.56 months) according to Uppal et al. [26], which confirms previous reports $[21,23]$. In the latter 


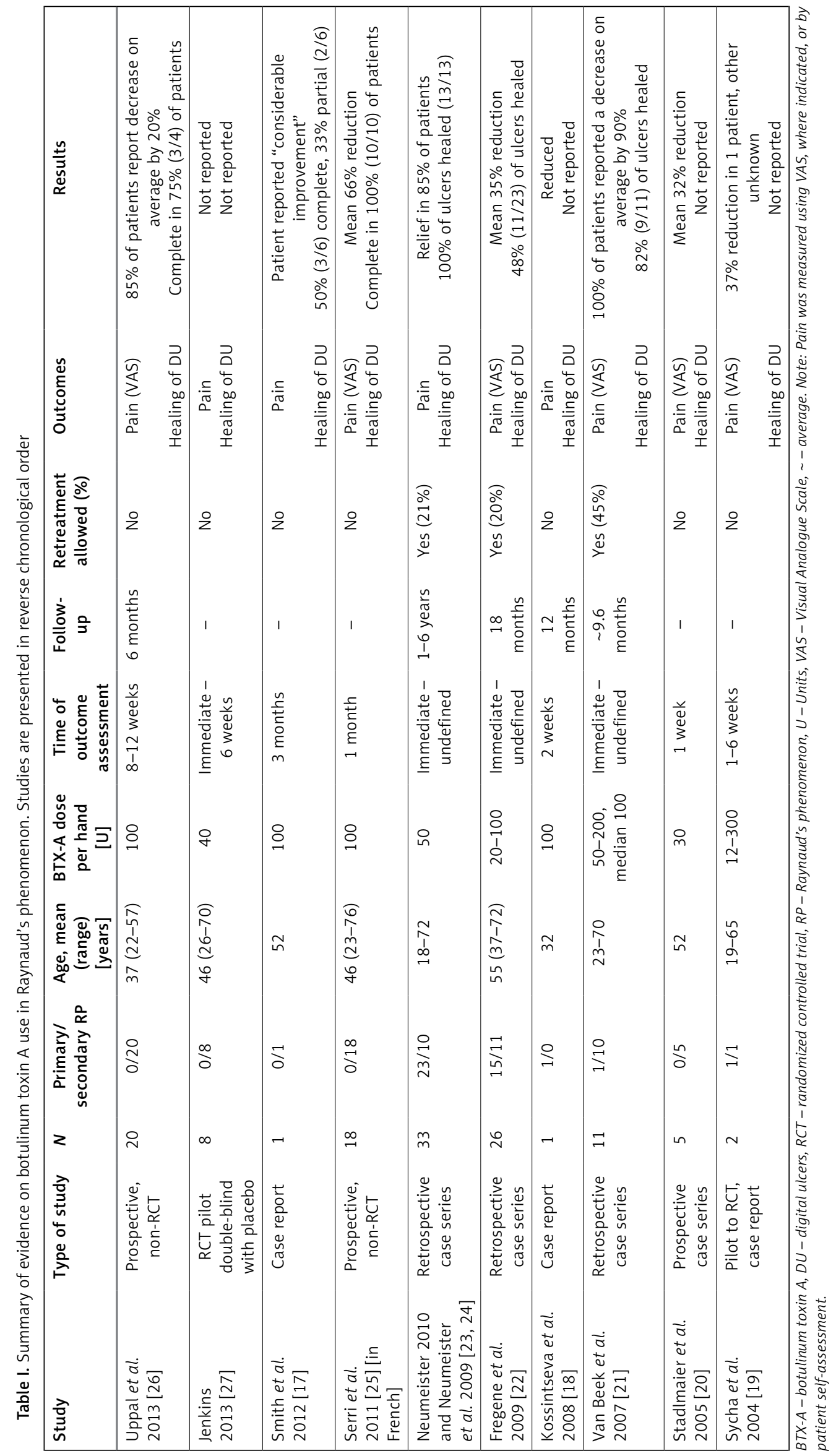


two studies, $21-45 \%$ of patients required retreatment due to relapse of RP symptoms, according to Van Beek et al. every 3-8 months [21].

The reported adverse events of BTX-A treatment were mild and transient. Injection-related pain was not reported beyond the treatment day [22]. Between $9 \%$ and $27 \%$ of patients suffered hand muscle weakness that resolved within 2-3 months $[21-23,25,26]$. There were no serious adverse events reported in the literature.

The only completed randomized controlled trial (RCT) pilot of BTX-A use in RP (ClinicalTrials. gov NCT01233999) was undertaken by Jenkins et al. [27]. Patients were randomized to receive BTX-A injections into either hand, while the contralateral hand was injected with saline as a control. A significant increase of digital pulp temperature was demonstrated with no difference in cold recovery times after 20 -second ice bath immersion. Pain and ulcer healing was not reported. We contacted the author of this study, asking whether it was going to be continued, and we were informed that it is unlikely in the foreseeable future.

A randomized double-blind placebo-controlled trial by Neumeister et al. registered at ClinicalTrials.gov (NCT01309802) evaluating BTX-A efficacy in RP is currently underway. The study involves patients with both primary $(n=20)$ and secondary $(n=20)$ RP randomly assigned to treatment with BTX-A (100 U per hand) or placebo. The primary endpoint is the number of pain-free days and pain intensity at baseline and 28 days after BTX-A injection. Secondary outcomes include: quality of life, hand function assessed by the QuickDASH score, patient satisfaction, tissue perfusion measured by LDPI and quality-adjusted life-years. The authors aim to follow up patients for up to 5 years to determine the long-term effects of BTX-A treatment including ulcer healing and the need for retreatment [28].

Another double-blind RCT evaluating the BTX-A effect on 40 secondary RP patients with systemic sclerosis is planned to start in September 2014 (NCT02165111). Its primary endpoint is blood flow to the fingers, as measured by non-invasive LDPI, whereas secondary endpoints include pain (VAS), healing of existing digital ulcerations and/ or prevention of new areas of digital ulceration, as well as assessment of RP's symptom severity using a number of scores (Raynaud's Condition Score, Quick-DASH, McCabe Cold Sensitivity) at periodic visits over a four-month period [29].

\section{Discussion}

The studies reviewed vary greatly in design and methodology. All studies consist of small groups of patients, lack standardization of injection sites as well as outcomes, and 7 out of 11 of them are retrospective. Thus the level of evidence across studies according to GRADE ranges from very low to moderate, as no large double-blind RCT has been completed so far. Nevertheless, all of them claim good efficacy and a highly acceptable safety profile of BTX-A injections for severe RP.

Despite being a lethal neurotoxin, BTX-A in clinical use has an excellent safety profile and few side effects. Immunologic phenomena such as urticaria or anaphylaxis are exceedingly rare. Unintended transient muscle paralysis and complications typical of any subcutaneous drug injection may occur [12].

The risk of bias in studies reviewed is high due to a lack of controls and a failure to ensure blinding of patients and health providers, except in the pilot RCT by Jenkins et al. [27]. We found no studies registered in either CENTRAL or ClinicalTrials.gov that were completed or stopped and not published, so publication bias is not likely.

There are also ethical concerns in designing RCTs. As Van Beek et al. pointed out, it would be unethical not to provide treatment that can potentially reverse ischemia and prevent autoamputation in patients with digital ulcers [21].

Although the studies follow different injection protocols, they all have the digital neurovascular bundle at one level or another as their target. Due to the heterogeneity and paucity of studies, there is insufficient evidence to determine whether the treatment effect of BTX-A is dose or injection-site related. An expert consensus on the optimal injection protocol associated with minimal side effects would benefit future studies on BTX-A in RP.

Some of the outcomes used by the researchers despite their objectivity (digital hand temperature, LDPI) are not clinically relevant. Research into the efficacy of BTX-A and other interventions in RP could be enhanced by the uniform application of clinically important endpoints, such as healing of digital ulcers, the length of the ulcer-free period, pain as measured using the VAS, and the length of the pain-free period. The criteria could be chosen using the Delphi method by an international group of experts associated with rheumatology societies such as the ACR (American College of Rheumatology) and EULAR (European League Against Rheumatism).

It is possible that some patients may have been incorrectly classified as having primary RP when in fact they had ischemic complications and therefore by definition should be diagnosed with secondary RP, possibly due to systemic sclerosis. Connective tissue diseases pose diagnostic challenges even to experienced rheumatologists; therefore other specialists performing BTX-A injections may not have been aware of the patients' underlying conditions. 
It is difficult to predict which patient groups would benefit most from the use of BTX-A. The reviewed studies focus on secondary RP patients with severe symptoms and frequent ischemic complications, in whom conservative pharmacologic and surgical methods have failed to control RP. In this group the need for optimal treatment is desperate. However, little is known about the potential use of BTX-A in primary RP, which is more of a nuisance than a serious disorder. Similarly, one may ask what is the appropriate place of therapy with BTX-A: as first line, second-line or add-on therapy. The reviewed studies use BTX-A as addon therapy except one case report [18], so we have no evidence on how onabotulinum could be used as a first or second line treatment modality.

The long-term efficacy of BTX-A should also be assessed in order to determine the frequency of need for re-treatment, as the effect is known to wane over time.

There is a pressing need for further research in this area, and there are sufficient data to justify conducting an RCT including large groups of patients with RP. Such a trial would provide the high-quality evidence that is necessary for clinical decision-making.

It is hoped that the ongoing study by Neumeister et al. in primary and secondary RP [28] and the study by Lifchez in secondary RP patients with systemic sclerosis [29] will provide answers about the clinical efficacy of BTX-A as well as its longterm effect on RP symptom control and trophic complications in respective groups of patients.

In conclusion, severe RP unresponsive to medical therapy is a significant problem for the patient and the rheumatologist. BTX-A injections, which are minimally invasive and have a low rate of complications, have the potential to become a breakthrough in the treatment of patients with severe RP. Although the results of the majority of studies of BTX-A efficacy in RP conducted so far were favorable, current evidence is not strong enough to either prove or disprove its efficacy. Therefore, new RCTs are needed in order to confidently assess this form of treatment.

\section{Acknowledgments}

This study was funded by a grant from Poznan University of Medical Sciences. The authors would like to express their gratitude to Jan Krzysztof Nowak $M D$, who helped in the review of the manuscript.

The authors declare no conflict of interest relevant to the contents of the article.

\section{References}

1. Herrick AL. The pathogenesis, diagnosis and treatment of Raynaud phenomenon. Nat Rev Rheumatol 2012; 8: 469-79.
2. Wigley FM. Clinical practice. Raynaud's phenomenon. N Engl J Med 2002; 347: 1001-8.

3. LeRoy EC, Medsger TA, Jr. Raynaud's phenomenon: a proposal for classification. Clin Exp Rheumatol 1992; 10: 485-8.

4. Riera G, Vilardell M, Vaque J, et al. Prevalence of Raynaud's phenomenon in a healthy Spanish population. J Rheumatol 1993; 20: 66-9.

5. Levien TL. Advances in the treatment of Raynaud's phenomenon. Vasc Health Risk Manag 2010; 6: 167-77.

6. Goundry B, Bell L, Langtree M, et al. Diagnosis and management of Raynaud's phenomenon. BMJ 2012; 344: e289.

7. Landry GJ. Current medical and surgical management of Raynaud's syndrome. J Vasc Surg 2013; 57: 1710-6.

8. Coveliers HME, Hoexum F, Nederhoed JH, et al. Thoracic sympathectomy for digital ischemia: a summary of evidence. J Vasc Surg 2011; 54: 273-7.

9. Herrick AL. Management of Raynaud's phenomenon and digital ischemia. Curr Rheumatol Rep 2013; 15: 303.

10. Botzoris V, Drosos AA. Management of Raynaud's phenomenon and digital ulcers in systemic sclerosis. Joint Bone Spine 2011; 78: 341-6.

11. Ennis H, Anderson ME, Wilkinson J, et al. Calcium channel blockers for primary Raynaud's phenomenon. Cochrane Database Syst Rev 2014; 1: CD002069.

12. Cartee TV, Monheit GD. An overview of botulinum toxins: past, present, and future. Clin Plast Surg 2011; 38: 409-26.

13. Stone AV, Koman LA, Callahan MF, et al. The effect of botulinum neurotoxin-A on blood flow in rats: a potential mechanism for treatment of Raynaud phenomenon. J Hand Surg Am 2012; 37: 795-802.

14. Naumann M, So Y, Argoff CE, et al. Assessment: Botulinum neurotoxin in the treatment of autonomic disorders and pain (an evidence-based review): report of the Therapeutics and Technology Assessment Subcommittee of the American Academy of Neurology. Neurology 2008; 70: 1707-14.

15. Iorio ML, Masden DL, Higgins JP. Botulinum toxin A treatment of Raynaud's phenomenon: a review. Semin Arthritis Rheum 2012; 41: 599-603.

16. Liberati A, Altman DG, Tetzlaff J, et al. The PRISMA statement for reporting systematic reviews and meta-analyses of studies that evaluate healthcare interventions: explanation and elaboration. BMJ 2009; 339: b2700.

17. Smith L, Polsky D, Franks AG Jr. Botulinum toxin-A for the treatment of Raynaud syndrome. Arch Dermatol 2012; 148: 426-8.

18. Kossintseva I, Barankin B. Improvement in both Raynaud disease and hyperhidrosis in response to botulinum toxin type A treatment. J Cutan Med Surg 2008; 12: 189-93.

19. Sycha T, Graninger M, Auff E, et al. Botulinum toxin in the treatment of Raynaud's phenomenon: a pilot study. Eur J Clin Invest 2004; 34: 312-3.

20. Stadlmaier E, Mueller T, Hermann J, Graninger WB. Raynaud's phenomenon: treatment with botulinum toxin. EULAR; Vienna, Austria. Ann Rheum Dis 2005; 64 (Suppl. III): 2752005.

21. Van Beek AL, Lim PK, Gear AJ, et al. Management of vasospastic disorders with botulinum toxin A. Plast Reconstr Surg 2007; 119: 217-26.

22. Fregene A, Ditmars D, Siddiqui A. Botulinum toxin type A: a treatment option for digital ischemia in patients with Raynaud's phenomenon. J Hand Surg Am 2009; 34: 446-52. 
23. Neumeister MW. Botulinum toxin type $A$ in the treatment of Raynaud's phenomenon. J Hand Surg Am 2010; 35: 2085-92.

24. Neumeister MW, Chambers CB, Herron MS, et al. Botox therapy for ischemic digits. Plast Reconstr Surg 2009; 124: 191-201.

25. Serri J, Legre R, Veit $V$, et al. Botulinum toxin type A contribution in the treatment of Raynaud's phenomenon due to systemic sclerosis. Ann Chir Plast Esthet 2013; 58: 658-62.

26. Uppal L, Dhaliwal K, Butler PE. A prospective study of the use of botulinum toxin injections in the treatment of Raynaud's syndrome associated with scleroderma. J Hand Surg Eur Vol 2014; 39: 876-80.

27. Jenkins SN, Neyman KM, Veledar E, et al. A pilot study evaluating the efficacy of botulinum toxin $A$ in the treatment of Raynaud phenomenon. J Am Acad Dermatol 2013; 69: 834-5.

28. Neumeister MW. A two-part study of BOTOX® therapy for ischemic digits: ClinicalTrials.gov Identifier: NCT01309802 [Last update: April 3, 2014; Date Last Accessed: July 29, 2014]. Available from: http://clinicaltrial.gov/ct2/show/record/NCT01309802.

29. Lifchez SD. Efficacy of botulinum toxin in scleroderma-associated Raynaud's syndrome: ClinicalTrials.gov Identifier: NCT02165111 [Last update: June 13, 2014; Date Last Accessed: July 29, 2014]. Available from: http:// clinicaltrials.gov/ct2/show/record/NCT02165111. 